\title{
Re: Dementia Assessment and Management Protocol for Doctors in Nepal
}

\author{
Martin Hofmeister ${ }^{1}$ \\ 'Consumer Centre of the German Federal State of Bavaria, Department Food and Nutrition, Munich, Germany.
}

Dear Editor,

I thank Jha and Sapkota for their interesting article, “Dementia assessment and management protocol for doctors in Nepal", published in a recent issue of the JNMA. ${ }^{1}$ I wish to make two additional comments regarding physical activity and exercise, both of which are essential for the prevention and treatment of dementia diseases.

Firstly, apart from dual-task deficits, motor and functional deficits are key early markers of dementia (e.g. ambulatory difficulties, limited forward flexion of the torso when getting up from a chair). ${ }^{2,3}$ A decline of functional and motor performances due to dementia is known to be the most common reason why elderly people develop a need for long-term care. In my opinion, validated functional performance tests such as the "Timed up-and-go test (TUG)" and "Five-times-sit-to-stand" test (FTSS) should therefore also be implemented as part of dementia assessment and the management protocol for Nepal. The test-retest reliability values for the quick and feasible TUG and FTSS have proven to be excellent or good, respectively, in older people with dementia. ${ }^{4}$ There is a negative association between each of the motor tools for assessing functional mobility or lower-limb strength and global cognitive performance. ${ }^{5}$

Secondly, in the context of post-diagnostic education and training protocol for dementia caregivers, the authors rightly pointed out that physical exercise is crucial for improving or at least maintaining motor performances and minimizing the risk of falling. ${ }^{1}$ Unfortunately, if they do so at all, patients tend to participate in training groups not adapted to the particular cognitive and motoric deficits of people with dementia. It would be desirable, if dementia caregivers in Nepal took into consideration that people with mild to moderate dementia could profit considerably from a progressive resistance and functional-balance training carried out twice weekly (e.g. walking, getting up from/sitting down on a chair, shifting of weight when standing, tiptoeing while holding on to the back of a chair, climbing stairs), combined with an individual dual-task training (e.g. walking while solving an arithmetic task such as counting backwards in increments of three). ${ }^{2,3}$ From a methodical point of view, training adjusted to dementia-related requirements should include simple, clearly structured exercises. These should be conveyed through dementia-specific verbal and nonverbal communication (e.g. brief instructions, positive wording, mirroring of movements, tactile support). ${ }^{3}$

Conflict of Interest: None.

Correspondence: Dr. Martin Hofmeister, Consumer Centre of the German Federal State of Bavaria Department Food and Nutrition, Munich, Germany. Email: hofmeister@vzbayern.de, Phone: $+4917661872779$ 


\section{REFERENCES}

1. Jha A, Sapkota N. Dementia assessment and management protocol for doctors in Nepal. JNMA J Nepal Med Assoc. 2013 Jan-Mar;52(189):292-8. [Full Text | PubMed]

2. Schwenk M, Zieschang T, Oster P, Hauer K. Dual-task performances can be improved in patients with dementia: a randomized controlled trial. Neurology. 2010 Jun 15;74(24):1961-8. [Full Text | PubMed]

3. Hauer K, Schwenk M, Zieschang T, Essig M, Becker C, Oster $\mathrm{P}$. Physical training improves motor performance in people with dementia: a randomized controlled trial. J Am Geriatr Soc. 2012 Jan;60(1):8-15. [Full Text | PubMed]
4. Blankevoort CG, van Heuvelen MJ, Scherder EJ. Reliability of six physical performance tests in older people with dementia. Phys Ther. 2013 Jan;93(1):69-78. [Full Text | PubMed]

5. Annweiler C, Schott AM, Abellan van Kan G, Rolland Y, Blain H, Fantino B, et al. The Five-Times-Sit-to-Stand test, a marker of global cognitive functioning among community-dwelling older women. J Nutr Health Aging. 2011 Apr;15(4):271-6. [Full Text | PubMed]

\section{AUTHORS' REPLY}

Re: Dementia assessment and management protocol for doctors in Nepal Arun Jha, ${ }^{1}$ Nidesh Sapkota ${ }^{2}$

${ }^{1}$ Mental Health services for Older People, Hertfordshire Partnership NHS Foundation Trust, ${ }^{2}$ Department of Psychiatry, BP Koirala Institute of Health Sciences, Dharan.

We very much appreciate for taking interest in our article, "Dementia assessment and management protocol for doctors in Nepal".

Your comments and suggestions are valid in preventing dementia, but there is not enough evidence for these intervention in treating dementia. However, they are useful as secondary preventive measures, especially in the context of vascular dementia.

We were unable to elaborate on these aspects as they were beyond the scope of the paper.

We would like to thank again for your observation. 